\title{
The fire wireless communication technology based on Mesh
}

\author{
XUE Caijiao \\ Basic Courses Teaching Department, The Chinese People's Armed Police Force Academy \\ Langfang, Hebei Province, China \\ Zhw_xcj@sina.com
}

Keywords:Fire; wireless communication; Mesh technology

\begin{abstract}
Fire wireless communications is an important guarantee for achieving fighting and rescue. The particularity of the fire rescue field needs higher request of the fire wireless communication. Considering Chinese fire wireless communication situation and the problem of fire site communication, Mesh wireless communication technology was proposed to resolve the problem. The article describes in detail the concept and characteristics of the Mesh network, designs the wireless communication network model on the Mesh technology, and conducts a detailed technical analysis. Then expound and prove the superiority of the fire wireless communication technology based on Mesh.
\end{abstract}

\section{Introduction}

With the rapid development of Chinese national economy, high-rise buildings, underground engineering, petrochemical companies, public gathering places are growing. Various types of fire accidents occur frequently, modern firefighting and rescue has been rendered more and more threedimensional, large space, large span situation. The fire and rescue scene particularity raised objectively new and high requirements of communication liaison between the fire commanders and command objects. The use of intelligent wireless networks for fire detection, fire real-time data acquisition, transmission of data, voice, image (multi-channel, multi-stream video) can protect fire smooth communication, develop disposal programs quickly, and correctly implement field command. How to quickly build a temporary intelligent wireless network at the fire scene has become an important issue.

\section{Fire Wireless Communications Status Quo}

After the actual research of the fire scene communication with the fire brigade the author discoveries several problems exist below:

- The number and type of individual communications, information gathering equipment is excessive. Advanced use of cluster network radio technology, fire communication helmets, digital phones, 3G data transmission systems, multi-carrier image transmission systems, POC audio communication systems, fire instruments transmission systems, electronic road water decision support systems, GPS satellite positioning systems bring great convenience to smooth fire communications. However, due to the lack of relevant standards and specifications, such systems were powered by a self-sufficient manner using separate data acquisition, information transmission network.

- Public network communication has disadvantages, easily affected by environmental constraints. The public mobile communications network was a relatively inexpensive mean of communication because it does not require the construction of the fire brigade. However, in addition to the problem of the existence of communications blackout, the main drawback is that it is difficult to meet the purpose of the fire and rescue scene in real-time communications. Because when the user increased dramatically, resource bandwidth is ideally trapped, communication base station is often paralyzed.

- It is difficult to coverage high-rise buildings or underground spaces because of the physical characteristics of electromagnetic waves. When the electromagnetic wave propagates in the 
space, the intensity of signal is attenuated with increasing transmission distance. Meanwhile, the different structures will produce different electromagnetic waves shielding effect, and therefore the use of a conventional single-hop network with the frequency signal is basically impossible to achieve full coverage of all buildings.

\section{Wireless Mesh Technologies}

Mesh technology is a new network technology in the wireless communications field at the late 1990s. Mesh (wireless mesh grid) technology can provide flexible and convenient communication in the absence of infrastructure support. It is a high capacity and high rate new distributed network technology. Mesh networks are also referred to as "multi-hop network" which can dynamically expand, and can provide effectively transmitting between wireless devices. It is a self-organizing and self-managed network because each node which has routing capabilities communicates only with adjacent nodes in the Mesh network. Mesh networks delivery subcontracting data from one route to another route until they reach their destination. These features make there is a significant difference between the Mesh network and the traditional cellular communications network.

In fact, Wireless mesh network (WMN) can be seen as a wireless version of the Internet. WMN is a new type of wireless broadband access network. It combines the advantages of the wireless local area network (WLAN) and Ad hoc network. It has own network, self-healing, multi-hop cascade, self-management nodes and other smart advantages. It has mobile broadband, wireless location, Internet access, and other features. It can extend traditional WLAN "hot spots" coverage to a wider range of "hot zone" covering.Figure.1 and Figure. 2 describe the structure of the two networks.

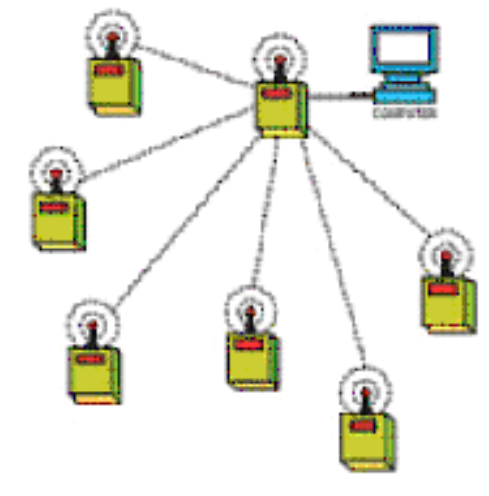

Figure.1 The traditional peer network

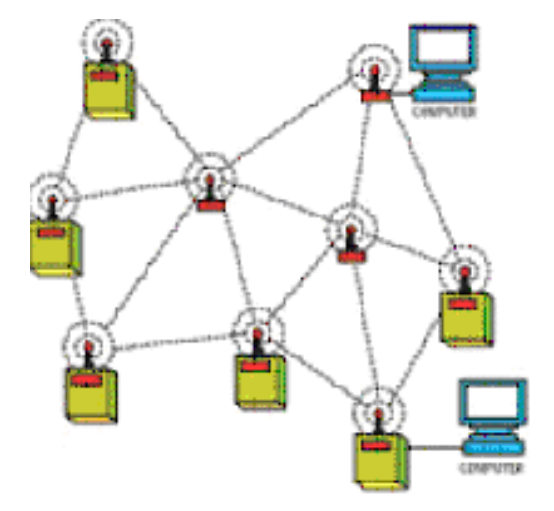

Figure.2 Wireless Mesh network

As can be seen from the Figures above:

- Compared with the traditional wireless network architecture, in wireless Mesh network, each node AP (Access Point) can communicate directly with one or more peer AP nodes. So at the same time a number of communication links can be smart choice for switching to improve the robustness of the network and optimize network links. 
- Wireless Mesh Networks has the advantages of low dependence on key nodes, expanding network coverage by relay ways. It is ideal for use in complex environments, especially for the underground tunnel and complex structure floor environment. We can quickly establish a communication link in a complex Wireless environment by relay mode using Wireless Mesh Network.

\section{Wireless Mesh Network Features}

Compared with traditional WLAN, Wireless Mesh Networks has several unparalleled advantages and features:

A. Rapid deployment and ease of installation

Mesh node installation is very simple. Take out the device from the packaging box, and connect the power on the line. Due to greatly simplify installation, the user can easily add new nodes to expand coverage and network capacity of the wireless network. In wireless Mesh network, not every Mesh node requires a wired cable connection, which is its biggest difference with the wired AP. Mesh design goal is minimizing the number of wired devices and wired APs. Thus it can greatly reduce the total cost of ownership and installation time. These cost saving is very considerable. Configuration and other network management functions of the wireless Mesh network are the same as the traditional WLAN. WLAN user's experience can be easily applied to the Mesh network.

\section{B. High Reliability}

The usual method of network robustness is to use multiple routers to transfer data. Mesh network is more robust than single-hop network, because it does not depend on a particular performance of a single node. In single-hop network, if one node fails, the entire network will be subsequently paralyzed. In the Mesh network structure, since each node has one or several data transmission paths. If a recent node is disturbed or fails, the packet will be automatically routed to an alternate path to continue transmission, and the operation of the entire network is not affected.

C. High bandwidth

Physical characteristics of wireless communication determine that the short-range communication transmission is easier to obtain a high-bandwidth than the long-range communication transmission, because with increasing wireless transmission distance, various interferences and other data loss factors will increase. So choosing multiple short hops to transmit data will be an effective way to achieve high network bandwidth, and this is what the advantage of the network mesh is. In the Mesh network, interconnecting with more nodes will increase the number of possible paths and increase greatly the total bandwidth.

D. High utilization

Since the transmission distance of each short hop is short, the power required to transmit data is also small. Since the multi-hop network typically uses less power to transmit data to an adjacent node, radio signal interference between nodes is small. Channel quality and channel utilization efficiency of the network is greatly improved, and then it is possible to achieve high network capacity. For example, in high-density urban network environment, Mesh network can reduce the wireless network interference of adjacent users, and greatly improve the utilization efficiency of the channel.

\section{Fire Wireless Communication Network Applications based on Mesh Technology}

\section{A. Networking}

There are some structure differences in WMN network and Mobile Ad hoc network. In general, depending on Character nodes in the WMN can be divided into client nodes, wireless Mesh router nodes and gateway nodes. Client nodes can be Wi-Fi phones, wireless sensors, radios, and other image acquisition devices; Mesh router nodes can be ordinary PCs, laptops, PDAs, and professional embedded systems. Ordinary WLAN client node is only general terminal equipment; Special Mesh client node not only has common terminal nodes access function, but also has routing and message forwarding functions. WMN network structure can be divided into Infrastructure network structure, 
Client network structure and Hybrid structure。

Infrastructure network structure provides their clients an infrastructure by Wireless Mesh router. There is a self-organizing, self-healing Mesh network formed between the Wireless Mesh Routers. Infrastructure network connects to the Internet by Mesh router gateway function, and provides the client Internet access services; Network node accesses the upper Mesh network structure through Mesh routers, and realizes the function of Internet communication. Infrastructure network structure is shown in Figure.3. Each communication terminal accesses the nearest mesh router.

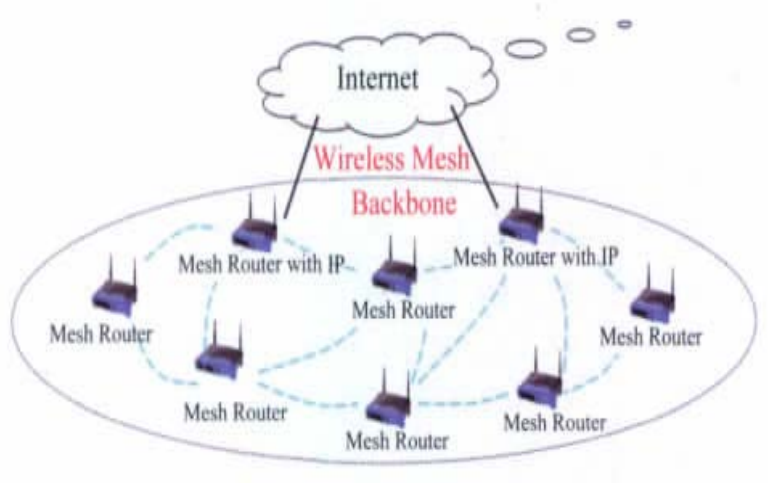

Figure.3 Infrastructure network structure

Client Mesh network structure (also called planar structure) is a point to point wireless channel network configured by the end user's own radios through wireless channel. Node can move, which could lead network topology change. Client network structure is in fact a mobile Ad hoc network. It can provide a communication support environment in the absence or the inconvenience use of existing network infrastructure. Although node is not required to have a gateway node and relay functions, node must have routing and self-organization capabilities.

In the hybrid structure, the terminal nodes increases Mesh device that has forwarding and routing functions. Devices can communicate each other directly by the Ad hoc way. Mesh clients can access the backbone network via Mesh routers. In general, the terminal node devices need to be able to support access to the upper Mesh networks and the peer nodes in this layer. This configuration provides number of other network structures connection, such as Internet, WLAN, WiMAX, cellular and sensor networks. At the same time, the client's routing capabilities can enhances connectivity, and expand coverage for WMN.

B. Networking process

Firstly, the fire brigade arrive the rescue scene, cover the entire site using fire communication and command vehicle, and quickly build a small wireless local area network of wireless Mesh technology.

Secondly, increase the antenna of command vehicle, so that LAN can establish a wireless connection with watchtowers, urban high-rise building nodes, or satellites. You can also let LAN access public security network (currently the majority of the country's fire brigade has set up public security network Line) to facilitate the data transmission by the wireless to cable equipment.

Finally, transmit fire image, data and other real-time information acquainted by firefighting vehicle to the command center using live fire image small wireless LAN. According to the site data in real time, the commander develops the right plan, and implements the firefighting and rescue. At the same time because the command vehicle is connected to the satellite, you can also locate the realtime fighting position of all vehicles through GPS which is important for commanding officers to put out the fire command.

C. Network Application Features

- The use of multi-mode wireless Mesh technology ensures the fire scene communication smooth at all the time. Because it can ensure fast recovery when a network failure, and it can expand the coverage ability.

- In the visual conditions, the distances of point to point connection is up to tens of meters, and the time of Mesh routing switching is less than one second. 
- In the visual conditions, when the emergency communications command vehicle moves speedily, the transfer rate is megabytes per second.

- The network transmission bandwidth is high, and the coverage is wide. The wireless coverage of Node is up to several hundred meters, and the wireless coverage can be increased to several kilometers by compatible QDMA protocol (Quad Division Multiple Access technology, is the combination of technology of a frequency-division multiple access, TDMA, CDMA, and multiple access protocol to avoid conflict with the carrier sense). Increasing power can increase the penetration capability, So you can also improve coverage by increasing power.

- The Mesh wireless network can be compatible with a variety of network protocols, such as 802.11b, 802.11g, etc.

- The Mesh wireless network can automatically switch terminals, can select optimal route. It has self-healing capability.

- The Mesh wireless network has strong network flexibility, because the warfighter can establish a communication route to establish a transmission link for complex environments (high-rise buildings, special material building, etc.) anywhere using carry equipment along with fire and rescue. Front-line combatants can easily send the image information inside disaster scene to the rear of the commander by Mesh Network to ensure commanders make the correct disposal program.

\section{Conclusions}

Rapid formation a wireless broadband network to transmit real-time dynamic information is critical for proper and timely critical command. Set up wireless networks using Mesh network technology temporarily can protect the commander's proper and timely implementation of the command according to information received. So the fire brigade performs firefighting and rescue missions at a reasonable and orderly situation by WNN.

\section{References}

[1] Xu Fang, Fan Yufeng. Mesh technology application in fire and rescue emergency communications [J]. Fire Science and Technology, 2014, 33 (4): 432-434.

[2] Zhou Jieying, Chan Fan, Lei Jun, Deng Yihui. Wireless mesh networking and application [J]. Laboratory Research and Exploration, 2011, 30 (12): 56-59.

[3] Yu Hai, Cao Lei. Wireless mesh (Mesh) networking technology based on WiFi[J]. Modern electronic technology, 2010,34 (10): 120-122.

[4] Wu Xiuqiang, MCA, Zhang Weidong, et al. A vehicle-mounted mesh network roaming anonymous access authentication protocol [J]. Computer Science, 2010, (2): 53-55.

[5] Zhu Xiping, FANG Xuming. Wireless network coding based multi-hop ad hoc network connectivity enhancement [J]. Southwest Jiao tong University, 2010, 57 (6): 972-976.

[6] Fan Yufeng, Xu Fang, Zhang Di. Fire wireless communication electromagnetic spectrum management research [J]. Fire Science and Technology, 2010, 29 (9): 797-799. 